\title{
Comment on mean residence time in flow systems
}

(Received 1 November 1983)

\section{Dear Sírs,}

Recentiy, Awasthi and Vasudeva [1] purported to show that the measured mean residence time for a flow system, $\vec{t}$, is not always equal to the quotient of the system's volume and the flow rate, $V / Q$. While the possibility that $\bar{t}<V / Q$ when the system has a stagnant (or "dead") zone has been extensively discussed in the literature, both physically and theoretically (see e.g. Levenspiel [2] and Rubinovitch and Mann [3]), the authors' suggestion that $\bar{t}>V / Q$ is new and is in contrast to a well-accepted result. Such a claim requires either a contrary theoretical argument or contrary experimental results. The latter must be obtained in exceptionally wellperformed and controlled experiments to assure their reliability. The authors do not provide any theoretical argument for their surprising result but rather base their conclusions on results which were obtained using questionable experimental and computational procedures.

A necessary, though not sufficient, control on residence time experiments is confirmation that the material balance is satisfied. That is, that the amount of tracer in the effluent stream is identical to the amount of tracer injected, $M$. This is expressed by

$$
M=Q \int_{0}^{\infty} c(t) \mathrm{d} t
$$

It has been shown that even very slight discrepancies in the material balance can cause serious error in the measured values of $t$ [4]. Unfortunately, the authors did not perform this test to assess the accuracy of their experimental and computational procedures.

Besides this major deficiency, there are several errors and questionable steps in the sample calculations given in the appendix. These also raise doubis about the validity of the results.

(1) The summations $\Sigma c$ and $\Sigma t c$ do not approximate the integrations $\int c \mathrm{~d} t$ and $\int t c \mathrm{~d} t$, respectively, unless the summations are multiplied by $\Delta t$. The inclusion of $\Delta t$ is not important when the ratio of the two sums is used to calculate $t$, but it is essential when the integrations of the tail of the tracer response curves is calculated by an analytical integral. The value of $\bar{t}$ in the appendix is incorrect since it was obtained by summing two incommensurable terms. The sumterms should be multiplied by $\Delta t=0.25 \mathrm{~min}$.

(2) The constant $W$ in the exponential function, that was uscd to approximate the tail was calculated from two adjacent time points, reported to at most two significant figures. This is imprecise. In addition, the value of $W$ given $(W=0.1178)$ does not follow from the numbers shown, which give $W=0.5341$.

(3) The integral expression on the top of the right column on p. 319 is incorrect; the term exp $\left(-W / t_{1}\right)$ multiplying the bracket should not appear there if the integration is performed correctly. Unfortunately, this term was used in calculating $\bar{t}$. The authors state that the tails contributed at most $5 \%$ to the integrations, but this will be up to $20 \%$ when the summations are reduced by a factor of 0.25 .

\section{Department of Chemical Engineering \\ University of Michigan}

RANE L. CURL.

Ann Arbor, MI 48109, U.S.A.

Department of Chemical Engineering

UZI MANN

Texas Technical University

Lubbock, TX 79409, U.S.A.

\section{REFERENCES}

[1] Awasthi R. C. and Vasudeva K., Chem. Engng Sci. 198338 313.

[2] Levenspiel O., Chemical Reaction Engineering, 2nd edn, Chapter 9. Wiley, New York 1972.

[3] Rubinovitch M. and Mann U., Chem. Engng Sci. 197934 1309.

[4] Curl R. L. and McMillan M. L., Amer. Inst. Chem. Engng J. 196612819 . 